\title{
Electro-Magnetic Velocity Meters: Assessment of the (Missing) Technical Parameters
}

\author{
Damjan Ivetic ${ }^{(凶)}$, Dusan Prodanovic, and Luka Stojadinovic \\ Faculty of Civil Engineering, University of Belgrade, Bul. kralja Aleksandra 73, \\ 11000 Belgrade, Serbia \\ \{divetic,dprodanovic\}@grf.bg.ac.rs, \\ st_luka@hotmail.com
}

\begin{abstract}
Flow measurements in Urban Drainage Systems are essential for the pollution control. Since the accuracy of the Acoustic Doppler Velocimeters is impeded by several factors, this research is focused on the alternative or a supplemental Electro-Magnetic Velocity (EMV) meters. EMV meters are more robust and reliable and can provide accurate measurements of low flows or even under a porous sediment cover. However, the downside of their usage is the small control volume $(\mathrm{CV})$ where the flow velocities are integrated in a nonlinear manner to obtain a single one-dimensional velocity measurement. To provide accurate mean flow velocity it is necessary to determine the size of the $\mathrm{CV}$ and to understand the non-linear integration principle within the CV. These valuable data are typically not provided by the manufacturer. In this paper, an experimental methodology is proposed for defining the size of the $\mathrm{CV}$ and the one-dimensional (1D) weighting function, used to describe the sensor's integration principle. In the experiments, bed-mounted flat DC2 EMV probe was used. The size of the CV and 1D weighting function were determined based on the results of the EMV operation under sand cover of varying depth. It was shown that the reach of the $\mathrm{CV}$ for the used device is $6 \mathrm{~cm}$ from the electrodes of the sensor.
\end{abstract}

Keywords: Flow measurements · Electro-Magnetic Velocity meter Weighting function

\section{Introduction}

Accurate flow measurements in Urban Drainage Systems (UDS) are essential for estimating pollutant loads and a better understanding of impacts on the urban aquatic environment. Flow measurements in UDS present a challenging task since they are designed to operate with partially filled pipes, commonly characterized by hostile environmental conditions. The Velocity-Area (VA) method with a bed-mounted Acoustic Doppler Velocimeters (ADV) are frequently used (Larrarte et al. 2008). However, it was shown that the ability of the ADV to provide accurate velocity measurements in UDS is impeded by several factors (Aguilar et al. 2016). Hence an investigation on alternative or a supplement sensor is needed. In this paper, the flat bedmounted Electro Magnetic Velocity (EMV) meter/sensor is analyzed. 
Due to its operating principle, the EMV meters are inherently more robust and reliable when compared to the ADV. It was shown that EMV meter can provide linear measurements of the flow velocity even under porous sediment cover of few centimeters (Ivetic et al. 2018 - in preparation). Additionally, EMV meter has good performance in flows with low depths (smaller than $5 \mathrm{~cm}$ ) and very low velocities (below $\mathrm{cm} / \mathrm{s}$ ), making it a valuable measuring device in the combined sewer systems where a dramatic difference is observed between dry and wet weather flows (Harramoes et al. 1993). The downside of the EMV meter is the small control volume (CV, flow volume contributing to the sensor's output signal), hence the velocity measurements are more "local" when compared to the ADV. Furthermore, velocity measurements are a result of the non-linear integration of the flow velocity distribution within the CV (Shercliff 1962). Hence, to provide accurate mean flow velocity measurements with the EMV sensor, it is necessary to know the size of the CV and to understand the velocity integration principle within the $\mathrm{CV}$. As the manufacturers of EMV meters are typically not providing the user with these valuable data, a suitable methodology for describing these features of the EMV meter is needed.

The focus of this research is to establish an experimental framework for defining the EMV's technical parameters, the size of the CV and the sensitivity of the measured velocity to the velocity distribution within the $\mathrm{CV}$. The aim is to improve the accuracy of the VA flow measurements in UDS, by including additional technical parameters of the EMV sensor in the measurement.

\section{Materials and Methods}

\section{$2.1 \quad$ Flat EMV}

In the research presented in this paper, a bed mounted flat DC2 EMV probe (Svet instrumenata 2012), designed by a local SME for one-dimensional velocity measurements, was used. Flat EMV probe is shaped to minimize the flow disturbances. Two flat excitation coils are integrated into the robust inox housing of the probe, with the dimensions of $\mathrm{L}=280 \mathrm{~mm}, \mathrm{~W}=160 \mathrm{~mm}$ and $\mathrm{H}=23 \mathrm{~mm}$ (Fig. 1A). The manufacturer specifies that the accuracy of the DC-2 EMV device is $\pm 1 \%$, precision $0.001 \mathrm{~m} / \mathrm{s}$, and the operating range $\pm 15 \mathrm{~m} / \mathrm{s}$. The induced voltage shows a linear relationship with the measured velocity.

In general, the EMV operating principle is based on the Faraday's law of induction, where the output signal (voltage $E$ ) is generated by the motion of the conductive fluid (water) through a transversal magnetic field (Shercliff 1962). Although the general sensitivity was described as the cross product of the velocity $\vec{V}$ and the magnetic field $\vec{B}$, Shercliff (1962) proposed a simple equation to describe how each part of the flow contributes to the voltage $E$ through the weight function $w$ :

$$
E=\int_{\tau} w \cdot \vec{V} d \tau
$$



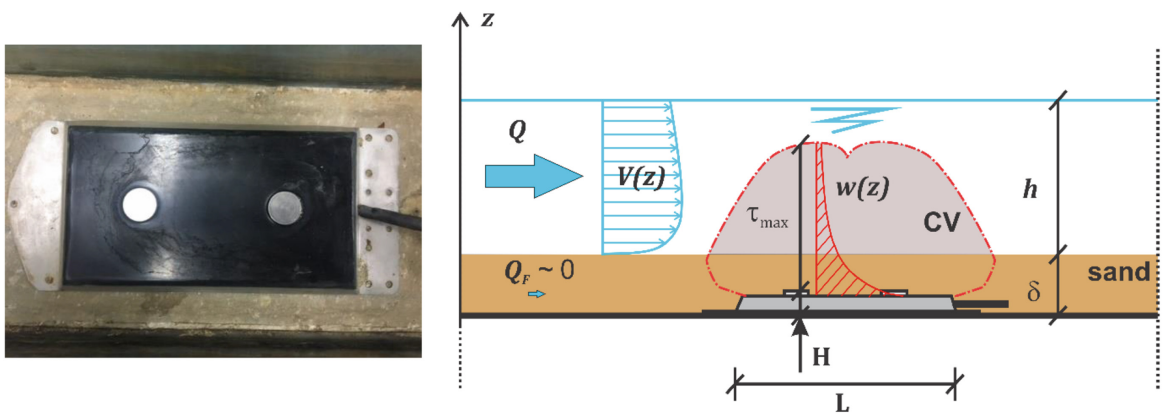

Fig. 1. Left (A): Top view of flat DC2 EMV meter in a lab flume; Right (B): Illustration of the Flat EMV operation under sand cover with parameters significant to the analysis

where $\tau$ is the control volume (CV) of the EM sensor (Fig. 1B). The CV is physically described as a volume (3D), yet for the practical purposes, it can be represented by a single parameter $\tau_{\max }$ - i.e. the reach of the $\mathrm{CV}$ or the upper limit of integration in Eq. (1).

In the bed-mounted EMV application, $\tau$ is a variable, dependent on several factors: excitation current, distribution of the $\vec{B}$, conduits geometry and water depth $h$ (when the his bellow $\tau_{\max }$, otherwise $\tau=\tau_{\max }$ ). Since the excitation coils of the flat EMV probe are relatively small and power consumption is kept low (coil excitation current $80 \mathrm{~mA}$ during the $10 \mathrm{~s}$ sampling period), the reach of the produced magnetic field is limited to the vicinity of the EMV (Fig. 1B). The goal of this research is to experimentally derive, one-dimensional $w$ and the maximum limits of the integration (the $\tau_{\max }$ ).

\subsection{Sand Cover Experiments}

To derive the weighing function, the relationship between the size of the CV and the EMV output voltage needs to be experimentally assessed. It is necessary to systematically exclude certain zones of the CV (in the $z$ direction) but still allow the contact between the conductive fluid and the electrodes (Fig. 1B). It is assumed that if the flat EMV is covered with the porous material (e.g. sand) of varying thickness $\delta$, this effect can be achieved. The zones occupied by sand, within the $\mathrm{CV}$, will not contribute to the output voltage as the filtration $\left(Q_{F}\right)$ velocity is negligible compared to the bulk flow $(Q)$ and the sand will not influence the magnetic field. The reduction of the CV in this manner, will lead to the proportionally smaller output voltage.

Previous flume experiments (Ivetic et al. 2018) have shown that the systematic effect of the sand cover, on the velocity measurements, can be minimized with the use of linear Correction Function Model (CFM), as long as the sand surface is flat. A part of the adapted lab flume, in the Faculty of Civil Engineering, University of Belgrade (Serbia), accommodating the free surface flow in an $8 \mathrm{~m}$ long and $0.25 \mathrm{~m}$ wide rectangular channel, has been used. Within these experiments, uncertainty benchmarking procedure (Aguilar et al. 2016) was utilized for assessing the velocity measurements acquired with the flat EMV under 16 different sand sediment depths $\delta=\{0,5,10,15$, 
$20,23,25,30,35,40,45,50,55,60,65,70,80 \mathrm{~mm}$. It was observed that the bias uncertainty increases with the increase of $\delta$ hence the parameters of the CFM model are dependant on the value of $\delta$. CFM is defined with two parameters, slope $\alpha$ representing the amplification of the output signal, and intercept $\beta$ corresponding to the zero-drift. Here, only the amplification of the signal $\alpha$ is considered, as the $\beta$ was proven to be constant for varying $\delta$. Based on the obtained CFM for sand, a power model for $\alpha$ (against $\delta$ ) was derived and plotted on Fig. 3A. The amplification $\alpha$ describes how the exclusion of the certain zones of the CV, starting from the EMV's upper surface $\left(z_{\text {sur }}\right)$, will reduce the strength of the output signal (Fig. 2A).
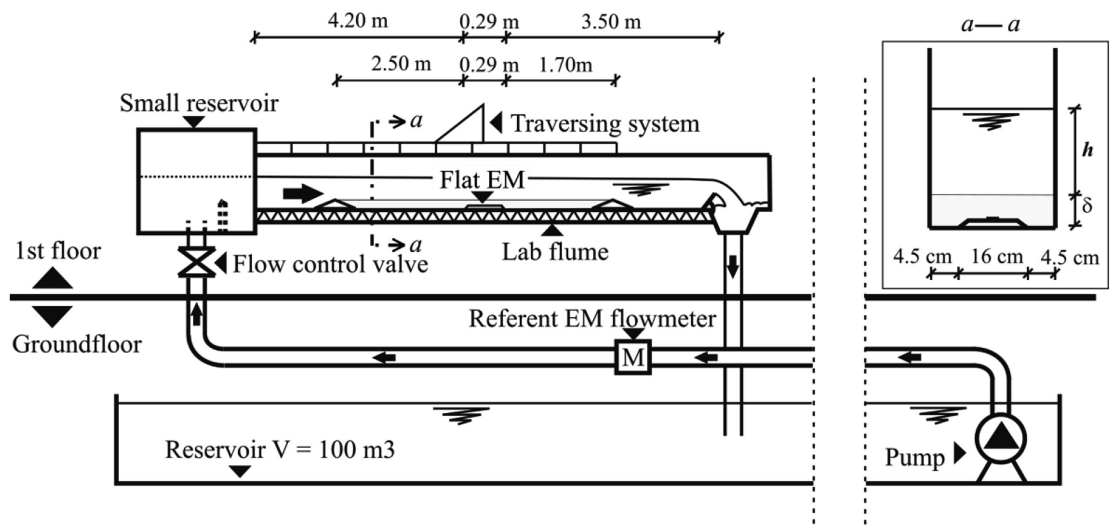

Fig. 2. A schematic illustration of the adapted laboratory flume setup as part of the closed circulation system

For defining the one-dimensional $w$, a vertical velocity distribution within the bulk flow is needed. As the upstream length of the sand cover in the experiments was over few meters (length/width $>10$ ), and the width/depth ratio was relatively low, the velocity distribution was approximated by the log-law extension proposed by Bonakdari et al. (2008). Next, it is necessary to discretise the vertical distance from $z_{\text {sur }}$ to $z_{\text {upp }}=z_{\text {sur }}+\tau_{\text {max }}$, by arbitrary $d z$, defining $i=1 \rightarrow N$ number of vertical distances $z_{i}=z_{u p p}+i d z$. Finally, for each of the $z_{i}$, numerically approximated Eq. (1), corresponding to the case of $\delta=z_{i}$, in form of a sum of squares can be written:

$$
N \alpha_{i} V=\sum_{i=1}^{N} w_{i} u_{i}
$$

where $V$ is the mean flow velocity. In total $N$ equations (2) can be defined, where for each equation $\alpha_{i}$ corresponds to the reduction of the $V$ for $\delta=z_{i}$. Furthermore, due to the presence of the sediment cover, for each of the $N$ equations (2) the distribution of the $u_{i}$ needs to be defined in accordance to the value $\delta=z_{i}$. 


\section{Results and Discussion}

The $N$ equations (2) form the system of linear equations with a lower diagonal matrix of unknown coefficients $w_{i}$ due to the fact that $Q_{F} \sim 0$. Solving this system leads to the experimentally defined one-dimensional weighting function $w$ (Fig. 3B). As $\tau_{\max }$ was not known a priori, it was varied until the smoothly receding $w$ was not computed. Finally, after verification with measured results, it was assumed that $\tau_{\max }$ is equal to $60 \mathrm{~mm}$.
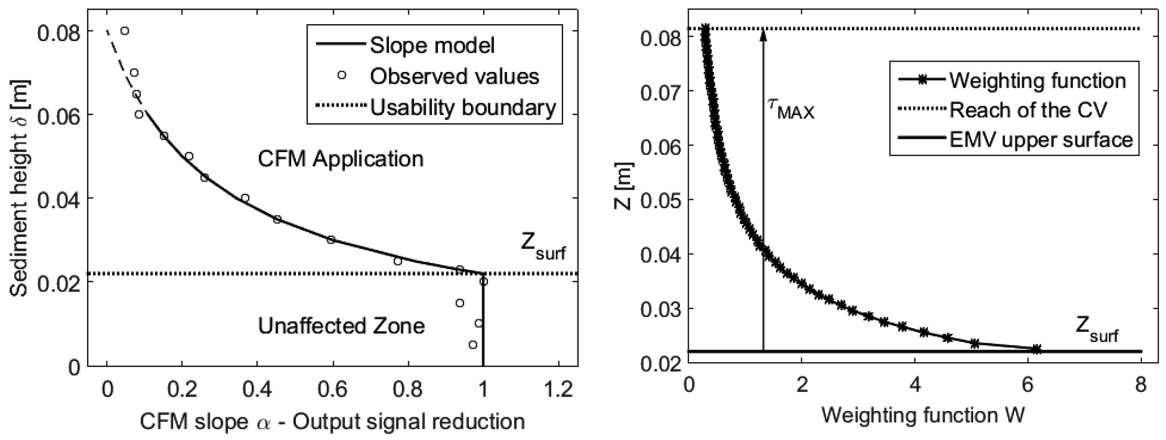

Fig. 3. Left (A): CFM slope model based on the "observed" values of the correction function slopes for varying sand cover height; Right (B): one-dimensional weighting function and the reach of the $\mathrm{CV}$, derived from the sand cover experiment

\section{Conclusions}

EMV meters can be considered as a supplement or an alternative to commonly used ADVs for velocity measurements in the UDS. Technical parameters, such as weighting function and the reach of the EMV meter's CV are essential for accurate mean velocity estimation. In this paper, a methodology for the experimental derivation of these parameters is given. For flat EMV meter, used in the experiments, it is shown that the control volume is limited to $6 \mathrm{~cm}$ from the sensor electrodes. Additionally, a corresponding one-dimensional weighting function is derived. With these technical parameters of the EMV meter, it is possible to improve the accuracy of the VA flow measurements in the UDS, based on the knowledge of the site-specific hydraulic and geometric characteristics. Further field investigations, probably supported by CFD analysis, are needed for assessing the potential for VA flow measurement improvement.

Acknowledgement. The authors are grateful to the Serbian Ministry of Education, Science and Technological Development for its financial support, project No. TR37010. 


\section{References}

Aguilar, M.F., McDonald, W.M., Dymond, R.L.: Benchmarking laboratory observation uncertainty for in-pipe storm sewer discharge measurements. J. Hydrol. 534, 73-86 (2016)

Bonakdari, H., Larrarte, F., Lassabatere, L., Joannis, C.: Turbulent velocity profile in fullydeveloped open channel flows. Environ. Fluid Mech. 8(1), 1-17 (2008)

Harremoës, P., Capodaglio, A.G., Hellström, B.G., Henze, M., Jensen, K.N., Lynggaard-Jensen, A., Otterpohl, R., Søeberg, H.: Wastewater treatment plants under transient loadingPerformance, modelling and control. Water Sci. Technol. 27(12), 71 (1993)

Ivetic, D., Prodanovic, D., Stojadinovic, L.: J. Hydrol. (2018, Submitted). HYDROL27705

Larrarte, F., Bardiaux, J.B., Battaglia, P., Joannis, C.: Acoustic doppler flow-meters: a proposal to characterize their technical parameters. Flow Meas. Instrum. 19(5), 261-267 (2008)

Shercliff, J.A.: The theory of electromagnetic flow-measurement. CUP Archive (1962)

Svet instrumenata. Flat DC2.34 EMV. Product Datasheet (2012, in Serbian). http://www.si.co.rs/ 